\title{
Aspects of Breast Cancer Between Two Different Societies
}

\author{
Salah E. Mostafa, MD, Maha Hemeida, MD, Abdul-Aziz Al-Nasser, MSC, MRCGP, Covin Jordon, MD* \\ Muslim M. Al-Saadi, Waleed A. Al-Ajroush.
}

DOI: http://dx.doi.org/10.5915/22-1-13523

\begin{abstract}
Breast cancer is the most common cancer among women in many countries. Mortality from the disease has increased steadily since the beginning of this century. This study explores the pattern of this disease in two different cultures, American and Saudi Arabian, and examines how culture can affect the pattern and risk factors for breast cancer. Breast cancer was found to be more common among younger Saudi women, 30-49 (69.4\%) and older American women (59.2\%). A positive family history was found in $11.4 \%$ and the incidence of recurrence was $16.6 \%$ among the Saudi Arabian group.
\end{abstract}

Key Words: Breast cancer, American group, Saudi Arabian group, age, site, family history, recurrence.

Cancer of the breast is the most commonly occurring cancer among women in industrialized countries. Mortality from the disease has been increasing steadily from beginning of this century.' Breast cancer accounts for one-fifth of all female deaths from cancer, and approximately $4 \%$ of deaths from all causes. It is the second most important cause of death among women aged 25-34 years, the leading cause among women aged 35-54, and second only to cardiovascular diseases at older age. ${ }^{2}$

The second most frequent malignancy among American women was found to be breast cancer, after lung cancer. ${ }^{3}$ Age over 40 years, previous family history of breast cancer, and previous breast cancer are some of the characteristics of high risk women. ${ }^{4}$

In blacks, breast cancer was diagnosed in a relatively more advanced stage than in whites, and

From the Department of Community and Family Medicine, King Saud University, P.O. Box 2925, \#34, Riyadh 11461, Saudi Arabia

*University of Mississippi, University and OxfordLafayatte County Hospital, Oxford, Mississippi, USA blacks have poorer survival rates.' Some findings suggest the possibility of involvement of seasonal factors acting at the fetal or neonatal stages in the etiology of breast cancer. Furthermore, the characteristics of patients with breast cancer differ in pre-menopausal women compared to postmenopausal women. ${ }^{6}$ Breast cancer is the commonest major malignant neoplasm in Saudi Arabian women.?

The incidence of radiation-induced breast cancer is the highest at the age of 76 years. "Some findings offer weak support for an association between weight or obesity and breast cancer risk, and suggest that anthropometric indices may be indirect indicators of true etiological factors for breast cancer.' It has been suggested that the risk of breast cancer is increased by oral contraceptive use before the first birth, or by the use before age 25 , particularly if certain pill formulations are used. ${ }^{10}$

The risk of breast cancer for a woman is higher if a first degree relative had unilateral (rather than bilateral) breast cancer detected at a young age." Breast cancer patients report a shorter average menstrual cycle length than those having benign breast disease or controls. ${ }^{12}$

Age at marriage (less than 20 years) and age at first pregnancies (less than 20 years) among Saudi women 


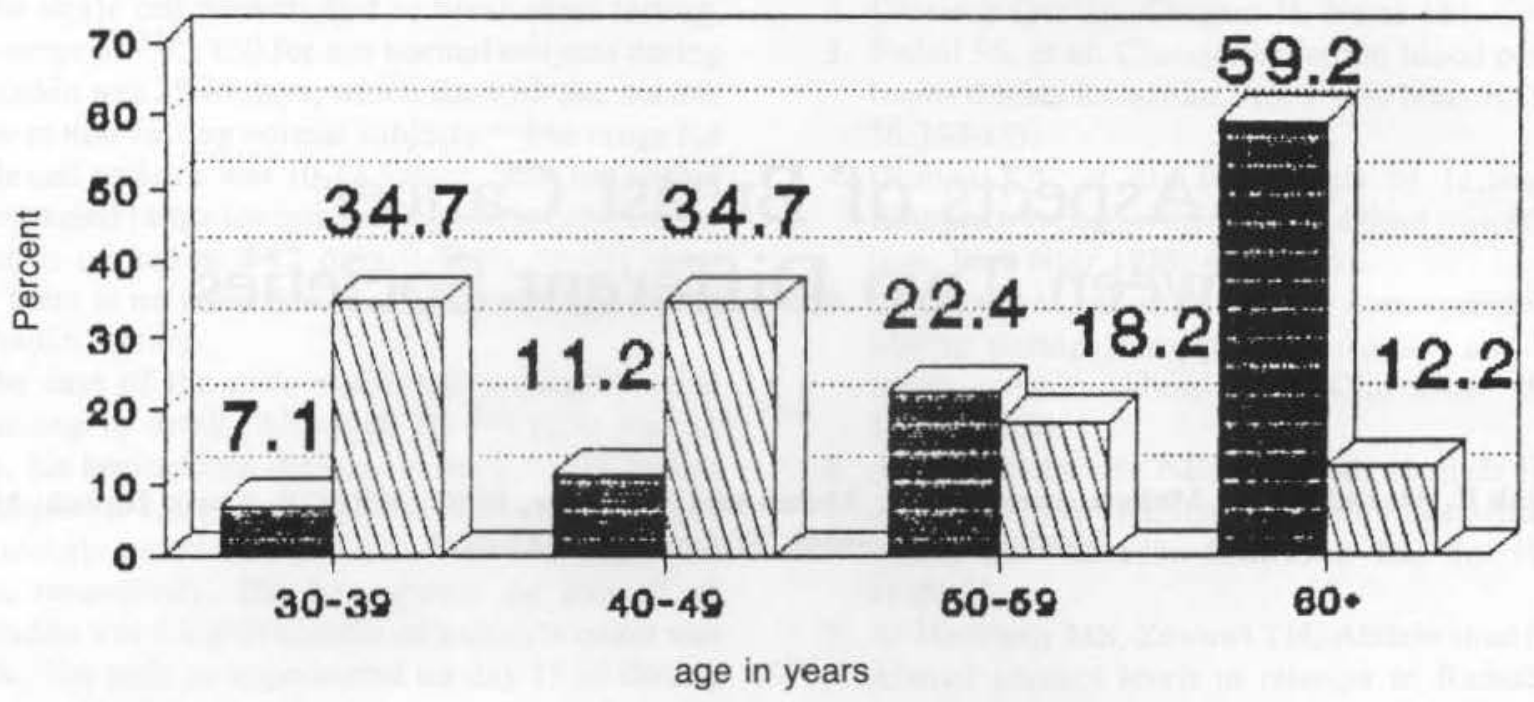

Damerlosn MU] saud

Figure 1: Breast cancer by age for both American and Saudi Women

Table 1. Frequency distribution of cases by age group for both American and Saudi groups.

\begin{tabular}{lrrrr}
\hline $\begin{array}{c}\text { Age } \\
\text { (years) }\end{array}$ & $\begin{array}{c}\text { American Group } \\
\text { n }\end{array}$ & \multicolumn{1}{c}{$\begin{array}{c}\text { Saudi Group } \\
\text { n }\end{array}$} & \multicolumn{1}{c}{$\%$} \\
\hline $30-39$ & 7 & 7.1 & 18 & 34.7 \\
$40-49$ & 11 & 11.2 & 17 & 34.7 \\
$50-59$ & 22 & 22.4 & 9 & 18.4 \\
$60+$ & 58 & 59.2 & 6 & 12.2 \\
Total & 98 & 99.9 & 50 & 100.0 \\
\hline
\end{tabular}

p 0.001

were $61.3 \%$ and $54.8 \%$ respectively. ${ }^{13}$ Parity (less than three and four or more children) among Saudi women were $53.3 \%$ and $46.7 \%$ respectively. ${ }^{14}$ Although birth control measures are available for Saudi women, no official statistical reports concerning their utilization could be found.

An early age at the first pregnancy has been generally acceptable as protective against the disease. ${ }^{15}$ The results of Moskowitz ${ }^{16}$ suggest that the most effective screening strategy may be mammography and clinical examinations for women aged 40-41 years, with biennial examinations thereafter.

The present study was done to compare the pattern of breast cancer in two different societies, Saudi Arabia and U.S.A., and to explore possible risk factors in both communities.

\section{Material and Methods}

Regarding Saudi data, the files of all breast cancer cases admitted to King Khalid University Hospital
Table 2. Frequency distribution of the site of carcinoma of the breast for both American and Saudi groups

\begin{tabular}{ccccc}
\hline $\begin{array}{c}\text { Site of } \\
\text { lesion }\end{array}$ & \multicolumn{2}{c}{ American Group } & \multicolumn{2}{c}{ Saudi Group } \\
$\mathbf{n}$ & $\%$ & $\mathbf{n}$ & $\%$ \\
\hline Right & 40 & 40.8 & 23 & 51.1 \\
Left & 58 & 59.2 & 22 & 48.9 \\
Total & 98 & 199.0 & $45^{*}$ & 100.0
\end{tabular}

*Data unavailable in 5 patients

(KKUH) within the years 1403-1407 H (1983-1987G) were abstracted. There were 50 female cases and one male case.

Data collected were age, nationality, sex, site of lesion, recurrence and family history. For the American data, 98 cases were collected from the cancer registry of a local community hospital in Oxford, Mississippi, during the years 1982-1985. The data collected included sex, race, age, and site of lesion. Chi square statistical tests were used in the analysis. The significance level was set at $\mathrm{p}=0.05$ for all tests.

\section{Results}

Table 1 and Figure 1 show that breast cancer is more common in younger Saudi women, i.e. in the $30-39$ age group $(34.7 \%)$ and the $40-49$ age group $(34.7 \%)$. On the other hand, breast cancer is more common in older American women, i.e. in the 50-59 age group $(22.4 \%)$ and the $60+$ age group $(59.2 \%)$. 
These differences are statistically significant at $p$ .001 .

The frequency distribution of the site of lesion for both American and Saudi groups are shown in Table 2. These data show that left-sided breast cancer occured in $59.2 \%$ of the American group but only $48.9 \%$ of Saudi women. The ratio of right to left among American women is $0.68: 1.00$ and among Saudi women 1.05:1.00.

Among 42 Saudi women for whom information is available, seven $(16.6 \%)$ had recurrences, five of which $(71.4 \%)$ occurred on the same side.

Among 44 Saudi women for whom information was available, only $5(11.4 \%)$ had positive family history. These data were unfortunately unavailable for the American group.

\section{Discussion}

Carcinoma of the breast is the most commonly occurring cancer of women in industrialized countries with increasing mortality, ${ }^{1}$ and it is the commonest major neoplasm in Saudi Arabian women. ${ }^{17,18}$

Donegan $^{19}$ showed that age at incidence curves in the majority of the developed countries demonstrate an increase in incidence throughout life, while in developing countries there appears to be an increasing incidence until middle age, then a plateau and gradual decline after the age of 50 years.

The results of this study show that most $(69.4 \%)$ of the Saudi cases occurred at a younger age, 30 to 50 years, while among the American group, the highest frequency of breast cancer was among women 50 years or older, at $81.6 \%$ (p 0.001 ). While this difference is similar to that described by Donegan, in Saudi women it may have other specific explanations.

In the last two decades Saudi Arabia has successfully developed medical facilities close to that which is available in many other western societies. These services are provided free of charges to its citizens. Although these medical facilities are available free of charge, there still is the possibility that some of the older Saudi women might not seek medical care as often or as regularly as younger women because of lack of education and customs. Moreover, a Saudi women has to find transportation to the medical facilities, due to cultural restrictions which may limit her chances to have suitable medical care. This is especially true for older women. The only way to determine whether the relatively low incidence of breast cancer among Saudi women is real or merely reflects under-reporting is to conduct a community survey to determine incidence, prevalence, and epidemiologial pattern of the disease.

Stirling et $\mathrm{al}^{20}$ reported that most Saudi women have their first child early in their reproductive life, and on the average have six pregnancies. Cancer of breast is by far the most common major malignancy in Saudi females, suggesting that the protective effect of early pregnancy and, to a lesser extent, multiple pregnancies is small.

Regarding side of lesion, our study showed that Americans have more lesions in the left side $(59.2 \%)$. This may support the results of Savlov ${ }^{21}$ who mentioned that the disease occurs more often on the left than on the right side, with ratio of 11 to 10 . There is almost no difference among the Saudi group (48.9\% left and $51.1 \%$ right).

It may be that the differences in pattern of breast cancer between the American and Saudi groups are related to the heterogeneous etiology of the disease in the two cultures. While the primary prevention of breast cancer is still beyond our reach, it has been shown beyond any doubt that cancer of the breast is amenable to secondary prevention. Secondary prevention includes breast self-examination, physical examination of the breast by physicians or paramedical personnel, and mammography.

\section{References}

1. Shimkin MB: Some Historical Landmarks in Cancer Epidemiology, Cancer Epidemiology and prevention, Current Concepts. Scholtenfeld D, ed. Springfield: C.C. Thomas, 1975.

2. Longan WPD: Cancer of breast - No decline in mortality. WHO Chronicle $1975 ; 29: 462-471$.

3. Surveillance, epidemiology, and end results: Incidence and mortality data, 1973-77. National Cancer Institute Monograph 57, NIH Publication No. 81-2330. Bethesda, Maryland: National Cancer Institute, 1981.

4. Thomas DB; Cancer. In: Last JM Chin J, Fielding JF, et al, eds, Maxcy-Rosenau Public Health and Preventive Medicine, 12th edition. Norwalk, Connecticut: Appleton-CenturyCrofts, 1986.

5. Nemoto J, Vana J, Bedwanirn R, et al: Management and survival of female breast cancer-Results of a national survey by the American College of Surgeons. Cancer 1980; 45:2917-2924.

6. Nakaoh S: Birth seasonality of breast cancer patients and its variation according to menopausal status and histologic type in Japan. Eur Cancer Clin Oncol 1986; 22:1105-1110.

7. El-Akkad S: Cancer in Saudi Arabia: A comparative study. Saudi Medical Journal 1983; 4:156.

8. Evans JS, Wemberg, JE, McNeil BJ: The influence of diagnostic radiography on the incidence of breast cancer and leukemia. N Engl J Med 1986; 315:810-815.

9. Kolonel LN, Nomura AM; Leel B: Anthropometric indications of breast cancer risk in 
post-menopausal women in Hawaii. Nutr Cancer $1986 ; 8: 247-256$.

10. Miller DR, Rosenberg L, Kaufuran DW, et al: Breast cancer risk in relation to early oral contraceptive use. Obstet Gyneol 1986; 68:863-868.

11. Sattin, RW, Rubin GL, Webster A, et al: Family history and the risk breast cancer. JAMA 1985; 233:1908-1913.

12. Olsson H, Landin OML, Gullberg B: Retrospective assessment of menstrual cycle length in patients with breast cancer and benign breast disease. INCI 1983; 70:17-20.

13. Mostafa S, Hemeida M: Aspects of a maternal child health center, in Riyadh area. Gaz Egypt Paed Ass 1988; 36:129-133.

14. Hemeida M, Mostafa S: Parity pattern among a group of mothers, Riyadh, Saudi Arabia 1988; $36: 37-42$.

15. Lynch HT, Albano WA, Layton MA, et al: Breast cancer genetics and age at first pregnancy.
J Med Genet 1984; 1:96-98.

16. Moskowitz M; Breast cancer age specific growth rates and screening strategies. Radiology 1986; 161:37-41.

17. Abdulla M, Gamal M, Aziz, A: Malignant neoplasms in Saudi Arabia. Cancer 1979; 44:1543.

18. Friedman RB, Harberg J: Cancer epidemiology, eight major sites. Wisconsin Med J 1978.

19. Donegan WL, Spratt JS: Cancer of the Breast. Philadelphia and London: WB Saunders Company, 1968.

20. Stirling GA, Khalil AM, Nada GN, et al: Study of one thousand consecutive malignant neoplasms in Saudis, 1975-1977. Saudi Medical J 1979; 1:89-99.

21. Savlov E: Breast Cancer, Clinical Oncology for Medical Students and Physicians: A Multidisciplinary Approach. Rubin P, ed. American Cancer Society, 3th ed, 1970-71. 